\section{New Theory}

ISSN: 2149-1402
36 (2021) 39-48

Journal of New Theory

https://dergipark.org.tr/en/pub/jnt

Open Access

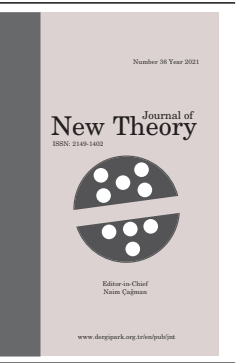

\title{
Roots of Second Order Polynomials with Real Coefficients in Elliptic Scator Algebra
}

\author{
Manuel Fernandez-Guasti ${ }^{1}$ (D)
}

\author{
Article History \\ Received: 23 Jun 2021 \\ Accepted: 18 Sep 2021 \\ Published: 30 Sep 2021 \\ 10.53570/jnt.956340 \\ Research Article
}

\begin{abstract}
The roots of second order polynomials with real coefficients are obtained in the $\mathbb{S}^{1+2}$ scator set. Explicit formulae are computed in terms of the polynomial coefficients. Although the scator product does not distribute over addition, the lack of distributivity is surmountable in order to find the zeros of the polynomial. The structure of the solutions and their distribution in $1+2$ dimensional scator space are illustrated and discussed. There exist six, two, or eight solutions, depending on the value of polynomial coefficients. Four of these roots only exist in the hypercomplex $\mathbb{S}^{1+2} \backslash \mathbb{S}^{1+1}$ set.
\end{abstract}

Keywords - Quadratic polynomial solutions, non-distributive algebras, hypercomplex numbers

Mathematics Subject Classification (2020) - 30G35, 20M14

\section{Introduction}

The quadratic equation $a x^{2}+b x+c=0, x \in \mathbb{H}$ in the quaternion set with real coefficients $a, b, c:$ i) if $4 a c \leq b^{2}$, has real solutions $x=\frac{1}{2 a}\left(-b \pm \sqrt{b^{2}-4 a c}\right)$ and ii) if $4 a c>b^{2}$, has an infinite number of solutions $x=-b+\beta \mathbf{i}+\gamma \mathbf{j}+\delta \mathbf{k}$, with $\beta^{2}+\gamma^{2}+\delta^{2}=4 c-b^{2}$ and $\beta, \gamma, \delta \in \mathbb{R}[1,2]$. In contrast, as we shall presently see, the quadratic equation with real coefficients in elliptic scator algebra has a finite number of roots.

Scator numbers are compound numbers that have one scalar component and $n$ director components in $\mathbb{R}^{1+n}, \stackrel{o}{\varphi}=f_{0}+\sum_{j=1}^{n} f_{j} \check{\mathbf{e}}_{j}$, where $f_{0}, f_{j} \in \mathbb{R}$ for $j$ from 1 to $n$ in $\mathbb{N}$ and $\check{\mathbf{e}}_{j} \notin \mathbb{R}$. Scator addition, performed component-wise, satisfies commutative group properties. Multiplication in elliptic scator algebra is commutative, possesses an identity element and all elements are invertible if zero is excluded. Multiplication is not associative if the additive scalar component of any two products vanish [3]. However, the non associative products can be isolated by imposing the appropriate conditions (i.e. $a_{j} b_{j} \neq a_{0} b_{0}$ in $\left.(3 \mathrm{a})\right)$, so that the additive scalar component of the products does not vanish. In general, scator multiplication is not distributive over addition [4]. Scator algebra has been successfully applied to several problems: time-space description in a deformed Lorentz metric [5], a wave-function evolution and collapse unified description in quantum mechanics [3] and three dimensional fractal structures [6]. Explicit formulae for scator holomorphic functions recently published, will very likely expand applications to other areas [7].

\footnotetext{
${ }^{1} \mathrm{mfg} @$ xanum.uam.mx (Corresponding Author)

${ }^{1}$ Laboratorio de Optica Cuantica, Departamento de Fisica, Universidad Autonoma Metropolitana - Iztapalapa, 09340 Ciudad de Mexico, AP 55-534, Mexico
} 


\subsection{Elliptic Scators}

Scator multiplication is defined in the scator set,

$$
\mathbb{S}^{1+n}=\left\{\stackrel{o}{\varphi}=f_{0}+\sum_{j=1}^{n} f_{j} \check{\mathbf{e}}_{j},\left(f_{0} ; \ldots f_{j}, \ldots f_{n}\right) \in \mathbb{R}^{1+n}: f_{0} \neq 0, \text { if there exists } f_{j} f_{l} \neq 0 \text { for } j \neq l \in n\right\}
$$

where the scalar component must be non-zero if two or more director components are non-zero. In particular, in $1+2$ dimensions,

$$
\mathbb{S}^{1+2}=\left\{\stackrel{o}{\varphi}=f_{0}+f_{1} \check{\mathbf{e}}_{1}+f_{2} \check{\mathbf{e}}_{2},\left(f_{0} ; f_{1}, f_{2}\right) \in \mathbb{R}^{1+2}: f_{0} \neq 0, \text { if } f_{1} f_{2} \neq 0\right\}
$$

The $\mathbb{S}_{j}^{1+1}$ scator set is isomorphic to the complex set $\mathbb{C}$ for the real component and any one of the $j$ director components. There are $n$ copies of the complex set embedded in $\mathbb{S}^{1+n}$ sharing the real part (named the scalar component in scator algebra) and having $n$ different hyper-imaginary parts. In this communication, we shall restrict to scators in $1+2$ dimensions.

Definition 1.1. The product of two scators $\stackrel{o}{\alpha}=a_{0}+a_{1} \check{\mathbf{e}}_{1}+a_{2} \check{\mathbf{e}}_{2} \in \mathbb{S}^{1+2}$ and $\stackrel{o}{\beta}=b_{0}+b_{1} \check{\mathbf{e}}_{1}+b_{2} \check{\mathbf{e}}_{2} \in \mathbb{S}^{1+2}$ is defined by:

If $a_{0} b_{0} \neq 0$,

$$
\stackrel{o}{\alpha \beta}=a_{0} b_{0}\left(1-\frac{a_{1} b_{1}}{a_{0} b_{0}}\right)\left(1-\frac{a_{2} b_{2}}{a_{0} b_{0}}\right)+\left(a_{0} b_{0}-a_{2} b_{2}\right)\left(\frac{a_{1}}{a_{0}}+\frac{b_{1}}{b_{0}}\right) \check{\mathbf{e}}_{1}+\left(a_{0} b_{0}-a_{1} b_{1}\right)\left(\frac{a_{2}}{a_{0}}+\frac{b_{2}}{b_{0}}\right) \check{\mathbf{e}}_{2}
$$

If $a_{0}=0$ and $b_{0} \neq 0$,

$$
\begin{aligned}
& \left(a_{1} \check{\mathbf{e}}_{1}\right) \stackrel{o}{\beta}=-a_{1} b_{1}+b_{0} a_{1} \check{\mathbf{e}}_{1}-\left(\frac{a_{1} b_{1} b_{2}}{b_{0}}\right) \check{\mathbf{e}}_{2} \\
& \left(a_{2} \check{\mathbf{e}}_{2}\right) \stackrel{o}{\beta}=-a_{2} b_{2}+b_{0} a_{2} \check{\mathbf{e}}_{2}-\left(\frac{a_{2} b_{2} b_{1}}{b_{0}}\right) \check{\mathbf{e}}_{1}
\end{aligned}
$$

If $a_{0}=b_{0}=0$,

$$
a_{1} \check{\mathbf{e}}_{1} b_{2} \check{\mathbf{e}}_{2}=0, \quad a_{1} \check{\mathbf{e}}_{1} b_{1} \check{\mathbf{e}}_{1}=-a_{1} b_{1}, \quad a_{2} \check{\mathbf{e}}_{2} b_{2} \check{\mathbf{e}}_{2}=-a_{2} b_{2}
$$

The conjugate of a scator $\stackrel{o}{\varphi}=f_{0}+f_{1} \check{\mathbf{e}}_{1}+f_{2} \check{\mathbf{e}}_{2} \in \mathbb{S}^{1+2}$ is obtained by reversing the sign of all the director components while leaving the scalar component unaltered, $\stackrel{o *}{\varphi}=f_{0}-f_{1} \check{\mathbf{e}}_{1}-f_{2} \check{\mathbf{e}}_{2}$. The magnitude of a scator $\left\|\varphi \varphi^{o}\right\| \in \mathbb{R}$, is given by the positive square root of the scator times its conjugate

$$
\|\stackrel{o}{\varphi}\|^{2}=\stackrel{o}{\varphi} \varphi^{*} * f_{0}^{2}\left(1+\frac{f_{1}^{2}}{f_{0}^{2}}\right)\left(1+\frac{f_{2}^{2}}{f_{0}^{2}}\right)
$$

if $f_{0} \neq 0$, and $\|\stackrel{o}{\varphi}\|^{2}=f_{j}^{2}$ if $f_{0}=0$.

\section{Roots of the Scator Quadratic Polynomial with Real Coefficients}

Consider the second order polynomial

$$
a \stackrel{o}{\varphi}^{2}+b \stackrel{o}{\varphi}+c=0
$$

where $\stackrel{o}{\varphi}$ is a scator element and $a, b, c \neq 0$ are real numbers. This polynomial cannot be factorized into $\left(\stackrel{o}{\varphi}-\stackrel{o}{r} r_{1}\right)\left(\stackrel{o}{\varphi}-\stackrel{o}{r_{2}}\right)=0$, since the scator product does not distribute over addition if $\stackrel{o}{\varphi} \in \mathbb{S}^{1+2} \backslash \mathbb{S}^{1+1}$. Furthermore, the product $\stackrel{\circ}{\alpha} \beta=0$ does not imply that $\stackrel{o}{\alpha}=0$ or $\stackrel{o}{\beta}=0$. The product of two scators $\stackrel{o}{\alpha}, \stackrel{o}{\beta}$ is zero if their components satisfy the conditions

$$
a_{0} b_{0}=a_{1} b_{1}=a_{2} b_{2}
$$


as may be seen from direct computation from the product definition (3a). For a given scator $\stackrel{o}{\alpha} \in$ $\mathbb{S}^{1+2} \backslash \mathbb{S}^{1+1}$, there always exists $\stackrel{o}{\beta}$ such that (6) is satisfied. Thus, all elements are zero divisors in the Bourbaki sense [8, p.98]. Nonetheless, it is possible to solve the polynomial equation in the scator domain without performing a factorization.

Theorem 2.1. The second order polynomial $a \stackrel{\stackrel{o}{\varphi}^{2}}{+}+b \stackrel{o}{\varphi}+c=0$, where $\stackrel{o}{\varphi} \in \mathbb{S}^{1+2}$ is an elliptic scator and $a, b, c \neq 0$ are real coefficients, has the following roots:

If $|4 a c|>b^{2}$, then

$$
\stackrel{o}{\varphi}_{\mathbb{S}^{1+2} \backslash \mathbb{S}^{1+1}}=-\frac{4 a c+b^{2}}{4 a b} \pm \sqrt{\frac{(4 a c)^{2}-\left(b^{2}\right)^{2}}{16 a^{2} b^{2}}} \check{\mathbf{e}}_{1} \pm \sqrt{\frac{(4 a c)^{2}-\left(b^{2}\right)^{2}}{16 a^{2} b^{2}}} \check{\mathbf{e}}_{2}
$$

If $4 a c \leq b^{2}$, then

$$
\stackrel{o}{\varphi}_{\mathbb{S}^{1+0}}=-\frac{b}{2 a} \pm \frac{\sqrt{b^{2}-4 a c}}{2 a}
$$

If $4 a c>b^{2}$, then

$$
\stackrel{o}{\varphi}_{\mathbb{S}_{1}^{1+1}}=-\frac{b}{2 a} \pm \frac{\sqrt{-b^{2}+4 a c}}{2 a} \check{\mathbf{e}}_{1}, \quad \stackrel{o}{\varphi}_{\mathbb{S}_{2}^{1+1}}=-\frac{b}{2 a} \pm \frac{\sqrt{-b^{2}+4 a c}}{2 a} \check{\mathbf{e}}_{2}
$$

Proof. Consider the scator $\stackrel{o}{\varphi}=f_{0}+f_{1} \check{\mathbf{e}}_{1}+f_{2} \check{\mathbf{e}}_{2} \in \mathbb{S}^{1+2} \backslash \mathbb{S}^{1+1}$, with non-vanishing components $f_{0}, f_{1}, f_{2} \neq 0$. From the product definition (3a), the square of this scator is,

$$
\stackrel{o}{\varphi}^{2}=f_{0}^{2}\left(1-\frac{f_{1}^{2}}{f_{0}^{2}}\right)\left(1-\frac{f_{2}^{2}}{f_{0}^{2}}\right)+2 f_{0} f_{1}\left(1-\frac{f_{2}^{2}}{f_{0}^{2}}\right) \check{\mathbf{e}}_{1}+2 f_{0} f_{2}\left(1-\frac{f_{1}^{2}}{f_{0}^{2}}\right) \check{\mathbf{e}}_{2}
$$

The polynomial (5), is then

$$
a\left[f_{0}^{2}\left(1-\frac{f_{1}^{2}}{f_{0}^{2}}\right)\left(1-\frac{f_{2}^{2}}{f_{0}^{2}}\right)+2 f_{0} f_{1}\left(1-\frac{f_{2}^{2}}{f_{0}^{2}}\right) \check{\mathbf{e}}_{1}+2 f_{0} f_{2}\left(1-\frac{f_{1}^{2}}{f_{0}^{2}}\right) \check{\mathbf{e}}_{2}\right]+b\left(f_{0}+f_{1} \check{\mathbf{e}}_{1}+f_{2} \check{\mathbf{e}}_{2}\right)+c=0
$$

Grouping components,

$a f_{0}^{2}\left(1-\frac{f_{1}^{2}}{f_{0}^{2}}\right)\left(1-\frac{f_{2}^{2}}{f_{0}^{2}}\right)+b f_{0}+c+\left(2 a f_{0} f_{1}\left(1-\frac{f_{2}^{2}}{f_{0}^{2}}\right)+b f_{1}\right) \check{\mathbf{e}}_{1}+\left(2 a f_{0} f_{2}\left(1-\frac{f_{1}^{2}}{f_{0}^{2}}\right)+b f_{2}\right) \check{\mathbf{e}}_{2}=0$

Two scators are equal if and only if all its additive components are equal. In particular, since the zero scator is $0=\stackrel{o}{0}=0+0 \check{\mathbf{e}}_{1}+0 \check{\mathbf{e}}_{2}$, the scalar component of the polynomial equation is then

$$
a f_{0}^{2}\left(1-\frac{f_{1}^{2}}{f_{0}^{2}}\right)\left(1-\frac{f_{2}^{2}}{f_{0}^{2}}\right)+b f_{0}+c=0
$$

whereas the director components equations are

$$
\begin{aligned}
& 2 a f_{0} f_{1}\left(1-\frac{f_{2}^{2}}{f_{0}^{2}}\right)+b f_{1}=0 \\
& 2 a f_{0} f_{2}\left(1-\frac{f_{1}^{2}}{f_{0}^{2}}\right)+b f_{2}=0
\end{aligned}
$$

Since $f_{1}$ and $f_{2}$ are both different from zero, Eq. (9b) can be multiplied by $\frac{1}{f_{1}}$ and (9c) by $\frac{1}{f_{2}}$, to obtain

$$
\begin{aligned}
& 2 a f_{0}\left(1-\frac{f_{2}^{2}}{f_{0}^{2}}\right)+b=0 \\
& 2 a f_{0}\left(1-\frac{f_{1}^{2}}{f_{0}^{2}}\right)+b=0
\end{aligned}
$$


The square of the two director components must then be equal, $f_{1}^{2}=f_{2}^{2}$. The term $1-\frac{f_{2}^{2}}{f_{0}^{2}}$ can be written in terms of $f_{0}$ from $(10 \mathrm{a})$ or $(10 \mathrm{~b}), 1-\frac{f_{2}^{2}}{f_{0}^{2}}=-\frac{b}{2 a f_{0}}$. The scalar component is then

$$
f_{0}=-\left(\frac{c}{b}+\frac{b}{4 a}\right)
$$

and the director components are

$$
f_{2}=f_{1}= \pm \sqrt{\frac{c^{2}}{b^{2}}-\frac{b^{2}}{16 a^{2}}}
$$

The four hyper-complex roots with non-vanishing director components are then

$$
\stackrel{o}{\varphi}_{\mathbb{S}^{1+2} \backslash \mathbb{S} 1+1}=-\left(\frac{c}{b}+\frac{b}{4 a}\right) \pm \sqrt{\frac{c^{2}}{b^{2}}-\frac{b^{2}}{16 a^{2}}} \check{\mathbf{e}}_{1} \pm \sqrt{\frac{c^{2}}{b^{2}}-\frac{b^{2}}{16 a^{2}}} \check{\mathbf{e}}_{2}
$$

These roots can be written in a similar form to the complex solutions as in equation (7a). To establish the interval where these roots exist, recall that the director coefficients were assumed to be non zero, thus $(4 a c)^{2} \neq\left(b^{2}\right)^{2}$. However, non-vanishing director coefficients also imply the inequality $(4 a c)^{2}>\left(b^{2}\right)^{2}$ : Assume $(4 a c)^{2}<\left(b^{2}\right)^{2}$, since the radicand is negative, $\sqrt{(4 a c)^{2}-\left(b^{2}\right)^{2}}=$ $\sqrt{\left(b^{2}\right)^{2}-(4 a c)^{2}} \sqrt{-1}$, where $\sqrt{\left(b^{2}\right)^{2}-(4 a c)^{2}} \in \mathbb{R}$. The root of minus one is any of the imaginary director units in scator algebra, let $\sqrt{-1}=\sqrt{\check{\mathbf{e}}_{1} \check{\mathbf{e}}_{1}}= \pm \check{\mathbf{e}}_{1}$. The first director term in (7a) is then $\sqrt{\frac{\left(\left(b^{2}\right)^{2}-(4 a c)^{2}\right)}{16 a^{2} b^{2}}} \check{\mathbf{e}}_{1} \check{\mathbf{e}}_{1}=-\sqrt{\frac{\left(\left(b^{2}\right)^{2}-(4 a c)^{2}\right)}{16 a^{2} b^{2}}} \in \mathbb{R}$, thus this director term is zero. For the second director term in $(7 \mathrm{a}), \sqrt{\frac{\left(\left(b^{2}\right)^{2}-(4 a c)^{2}\right)}{16 a^{2} b^{2}}} \check{\mathbf{e}}_{1} \check{\mathbf{e}}_{2}=0$, the director term is again zero. Therefore the two director coefficients vanish for $(4 a c)^{2}<\left(b^{2}\right)^{2}$.

If one of the director components is zero, then $\stackrel{o}{\varphi}=f_{0}+f_{1} \check{\mathbf{e}}_{1} \in \mathbb{S}_{1}^{1+1}$ or $\stackrel{o}{\varphi}=f_{0}+f_{2} \check{\mathbf{e}}_{2} \in \mathbb{S}_{2}^{1+1}$. If $f_{2}=0$, the polynomial ( 8 ) is

$$
a\left[\left(f_{0}^{2}-f_{1}^{2}\right)+2 f_{0} f_{1} \check{\mathbf{e}}_{1}\right]+b\left(f_{0}+f_{1} \check{\mathbf{e}}_{1}\right)+c=0
$$

and the real and $\check{\mathbf{e}}_{1}$ equations are $a\left(f_{0}^{2}-f_{1}^{2}\right)+b f_{0}+c=0$, and $2 f_{0} f_{1}+b f_{1}=0$, respectively. If $f_{1} \neq 0, f_{0}=-\frac{b}{2 a}$, the solutions if $4 a c>b^{2}$ are then,

$$
\stackrel{o}{\varphi}_{\mathbb{S}_{1}^{1+1}}=f_{0}+f_{1} \check{\mathbf{e}}_{1}=-\frac{b}{2 a} \pm \frac{\sqrt{-b^{2}+4 a c}}{2 a} \check{\mathbf{e}}_{1}
$$

If $4 a c \leq b^{2}, \sqrt{-b^{2}+4 a c}=\sqrt{b^{2}-4 a c} \check{\mathbf{e}}_{1}$, and thus

$$
\stackrel{o}{\varphi}_{\mathbb{S}_{1}^{1+1}}=-\frac{b}{2 a} \mp \frac{\sqrt{b^{2}-4 a c}}{2 a} \in \mathbb{S}^{1+0}
$$

Similarly, if $f_{1}=0$ and $4 a c>b^{2}$, then

$$
\stackrel{o}{\varphi}_{\mathbb{S}_{2}^{1+1}}=f_{0}+f_{2} \check{\mathbf{e}}_{2}=-\frac{b}{2 a} \pm \frac{\sqrt{-b^{2}+4 a c}}{2 a} \check{\mathbf{e}}_{2}
$$

whereas if $4 a c \leq b^{2}, \sqrt{-b^{2}+4 a c}=\sqrt{b^{2}-4 a c} \check{\mathbf{e}}_{2}$, thus

$$
\stackrel{o}{\varphi}_{\mathbb{S}_{2}^{1+1}}=-\frac{b}{2 a} \mp \frac{\sqrt{b^{2}-4 a c}}{2 a} \in \mathbb{S}^{1+0} .
$$

If the solutions are real, $\mathbb{S}^{1+0} \in\left(\mathbb{S}_{1}^{1+1} \cap \mathbb{S}_{2}^{1+1}\right)=\mathbb{S}^{1+0}$ This exhausts all possibilities stated in the proposition. 
We refer to solutions of the form (7a), as the hypercomplex roots. Notice that the scalar component of the solution is zero for the hypercomplex roots (7a) if $4 a c=-b^{2}$, but then the two director components are also zero. Thus, $\stackrel{o}{\varphi}_{\mathbb{S}^{1+2} \backslash \mathbb{S}^{1+1}} \in \mathbb{S}^{1+2}$. However, the hypercomplex roots are not a solution if $4 a c=-b^{2}$, the only solution is then given by the $\stackrel{o}{\varphi} \mathbb{S}^{1+0}$ real root. $\stackrel{o}{\varphi}$ is always in the $\mathbb{S}^{1+2}$ set, where the scator product is defined. Note that two of the conditions $|4 a c|>b^{2}, 4 a c \leq b^{2}$ and $4 a c>b^{2}$ can be met simultaneously depending on the values of $a, b$ and $c$, so that the hypercomplex roots can coexist with the real or complex roots.

In order to establish the interval where the hypercomplex roots $(7 \mathrm{a})$ exist, $\sqrt{-1}$ was written as $\check{\mathbf{e}}_{1}$ in the Theorem's proof. It could have equally been written as $\check{\mathbf{e}}_{2}$, then the product with $\check{\mathbf{e}}_{1}$ vanishes. As far as the proof is concerned, in both cases, the director component is zero, so no problems arise. However, the two results are not equal, i.e. $-1 \cdot \check{\mathbf{e}}_{1}=\left(\check{\mathbf{e}}_{1} \check{\mathbf{e}}_{1}\right) \check{\mathbf{e}}_{1}=-\check{\mathbf{e}}_{1}$, whereas $-1 \cdot \check{\mathbf{e}}_{1}=\left(\check{\mathbf{e}}_{2} \check{\mathbf{e}}_{2}\right) \check{\mathbf{e}}_{1} \neq \check{\mathbf{e}}_{2}\left(\check{\mathbf{e}}_{2} \check{\mathbf{e}}_{1}\right)=0$. The reason being that associativity does not hold when the product of two factors have zero scalar component, as is the case for $\left(\check{\mathbf{e}}_{2} \check{\mathbf{e}}_{1}\right)=0$.

It is reassuring to confirm that the solutions satisfy the polynomial equation. The real and complex like expressions are the familiar solutions. Let us evaluate the entirely novel hypercomplex solution (7a). The squared term for equal director components, from (3a) is

$$
\stackrel{o}{\varphi}^{2}=f_{0}^{2}\left(1-\frac{f_{1}^{2}}{f_{0}^{2}}\right)^{2}+2 f_{0} f_{1}\left(1-\frac{f_{1}^{2}}{f_{0}^{2}}\right) \check{\mathbf{e}}_{1}+2 f_{0} f_{1}\left(1-\frac{f_{1}^{2}}{f_{0}^{2}}\right) \check{\mathbf{e}}_{2}
$$

Evaluation of $f_{0}, f_{1}$ and $f_{2}$ from $(7 \mathrm{a})$, noting that $f_{0}\left(1-\frac{f_{1}^{2}}{f_{0}^{2}}\right)=-\frac{b}{2 a}$, after some algebra gives,

$$
\stackrel{o^{2}}{\mathbb{S}^{1+2} \backslash \mathbb{S}^{1+1}}=\frac{b^{2}}{4 a^{2}} \mp \frac{b}{a} \sqrt{\frac{(4 a c)^{2}-\left(b^{2}\right)^{2}}{16 a^{2} b^{2}}} \check{\mathbf{e}}_{1} \mp \frac{b}{a} \sqrt{\frac{(4 a c)^{2}-\left(b^{2}\right)^{2}}{16 a^{2} b^{2}}} \check{\mathbf{e}}_{2}
$$

The sum of $a$ times this expression plus $b \stackrel{o}{\varphi}_{\mathbb{S}^{1+2} \backslash \mathbb{S}^{1+1}}+c$, confirms that the quadratic polynomial equation (5) is satisfied.

\section{Geometric Representation of the Scator Roots in 1+2 Space}

If $4 a c \leq b^{2}$ and $|4 a c|>b^{2}$, there exist six roots: i) two real roots and ii) four hypercomplex roots. The initial conditions imply that $4 a c$ is negative, $4 a c+b^{2}<0$. Recalling that the hypercomplex scalar component is $-\frac{4 a c+b^{2}}{4 a b}$, these hypercomplex roots always lie in the real positive semispace if the product $a b$ is positive and in the real negative semispace if $a b$ is negative. These roots are depicted in Figure 1 , for $a=1, b=1, c=-1$. The two real roots (drawn in blue) are ${\stackrel{o}{\varphi^{1}+0}}=-\frac{1}{2} \pm \frac{\sqrt{5}}{2}$, whereas the four hypercomplex roots (drawn in orange) are $\stackrel{o}{\varphi}_{\mathbb{S}^{1+2} \backslash \mathbb{S}^{1+1}}=\frac{3}{4} \pm \frac{\sqrt{15}}{4} \check{\mathbf{e}}_{1} \pm \frac{\sqrt{15}}{4} \check{\mathbf{e}}_{2}$. It is interesting to notice that the hypercomplex roots coexist with real roots in this region.

If $4 a c>b^{2}$, there are eight roots because conditions $|4 a c|>b^{2}$ and $4 a c>b^{2}$ in Theorem 2.1

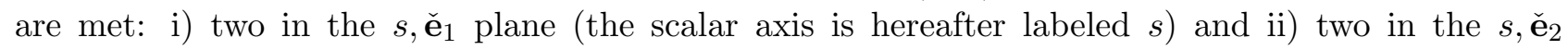
plane, corresponding to the complex roots in $\mathbb{C}$, but the imaginary parts are now the orthogonal $\check{\mathbf{e}}_{1}$, $\check{\mathbf{e}}_{2}$ imaginary units. iii) four hypercomplex roots that lie in the negative $s$ semispace if $a b$ is positive and in the real positive $s$ semispace if $a b$ is negative. These roots are shown in Figure 1, for $a=1, b=1, c=1$. The complex akin roots are $\stackrel{o}{\varphi}_{\mathbb{S}_{1}^{1+1}}=-\frac{1}{2} \pm \frac{\sqrt{3}}{2} \check{\mathbf{e}}_{1}$ (green) and $\stackrel{o}{\varphi}_{\mathbb{S}_{2}^{1+1}}=-\frac{1}{2} \pm \frac{\sqrt{3}}{2} \check{\mathbf{e}}_{2}$ (brown). The hypercomplex roots are $\stackrel{o}{\varphi}_{\mathbb{S}^{1+2} \backslash \mathbb{S}^{1+1}}=-\frac{5}{4} \pm \frac{\sqrt{15}}{4} \check{\mathbf{e}}_{1} \pm \frac{\sqrt{15}}{4} \check{\mathbf{e}}_{2}$ (drawn in red).

In the interval $-b^{2}<4 a c \leq b^{2}$, only the two real roots exist; these roots collapse to the same $-\frac{b}{2 a}$ value when $4 a c=b^{2}$. The regions where the different roots coexist are illustrated in Figure 2. Since the hypercomplex roots have directors with equal square components $f_{1}^{2}=f_{2}^{2}$, the hypercomplex roots always lie in planes at $45^{\circ}$ with respect to the $\check{\mathbf{e}}_{1}, \check{\mathbf{e}}_{2}$ axes. Let the function $f(a, b, c)=a \stackrel{o}{\varphi}^{2}+b \stackrel{o}{\varphi}+c$. The values of $\stackrel{o}{\varphi}$ where $f(a, b, c)$ is zero as a function of $c$, for constant $a, b$ are shown in Figure 3 . The possible values for the real, complex like and hypercomplex roots are shown in different colors. The 

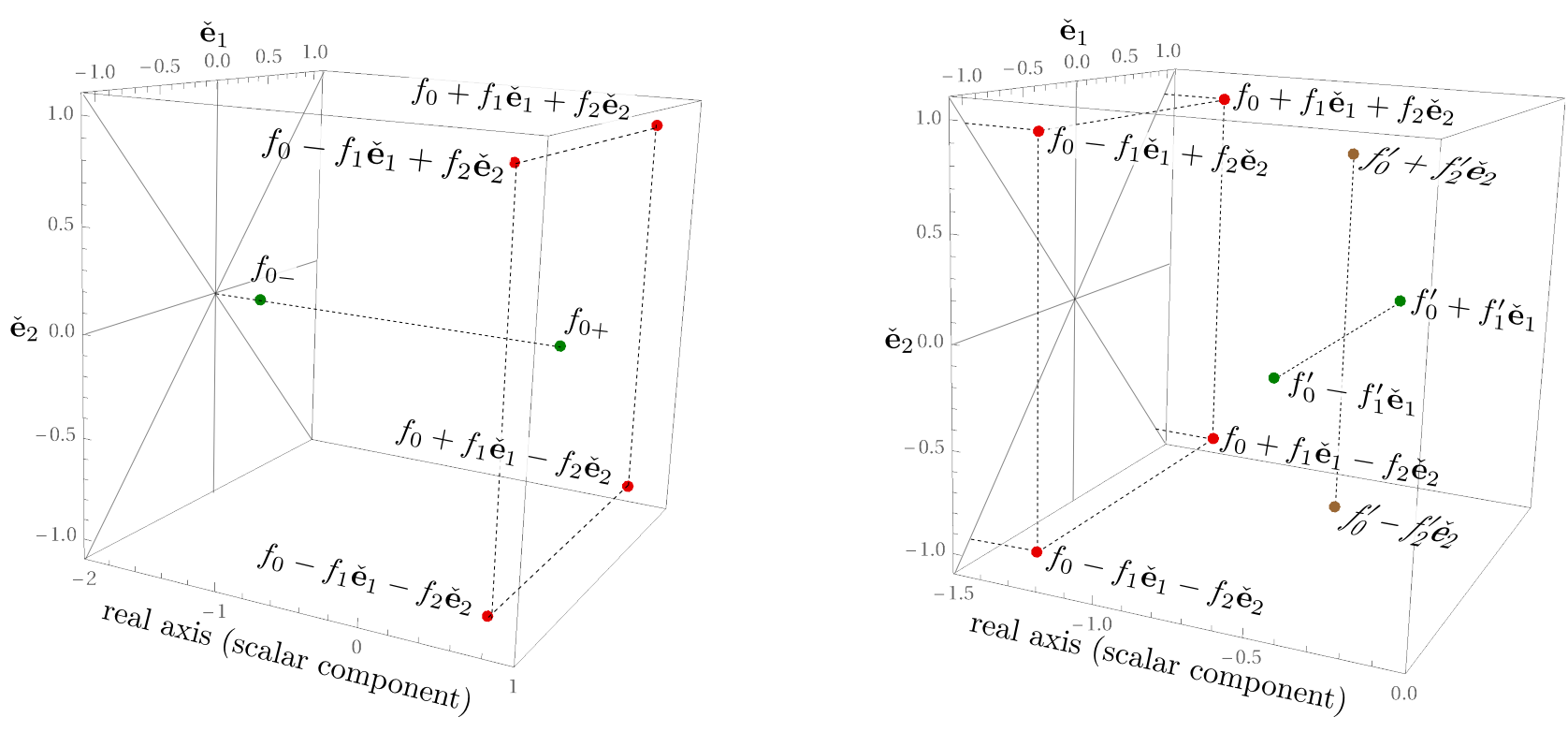

Fig. 1. Roots of the scator quadratic polynomial. Left: $4 a c \leq b^{2}$ and $|4 a c|>b^{2}$ with $a=1, b=$ $1, c=-1$. Hypercomplex roots in orange; real roots in blue. Right: $4 a c>b^{2}$ with $a=1, b=1, c=1$. Hypercomplex roots in red; complex like roots in the $s$, $\check{\mathbf{e}}_{1}$ plane in green and in the $s$, $\check{\mathbf{e}}_{2}$ plane in brown.

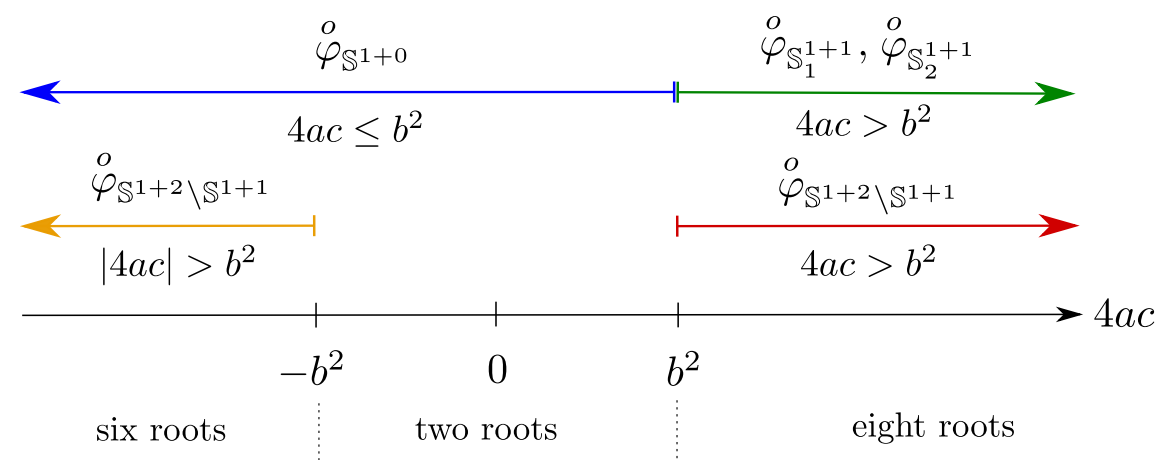

Fig. 2. The scator quadratic equation with real coefficients has six, two or eight roots, depending on the value of $4 a c$.

complex like roots (in green) have constant scalar component since the real (or scalar) part does not depend on $c$. There are however, two perpendicular branches, one for each hypercomplex direction $\check{\mathbf{e}}_{1}$ or $\check{\mathbf{e}}_{2}$. The hypercomplex roots with two nonvanishing director components, have been drawn in orange when they coexist with the real (blue) solutions and in red, when they coexist with the complex (green) solutions.

Lemma 3.1. Given a hypercomplex solution $\stackrel{o}{\varphi_{\mathbb{S}^{1+2}} \backslash \mathbb{S}^{1+1}}=f_{0}+f_{1} \check{\mathbf{e}}_{1}+f_{2} \check{\mathbf{e}}_{2}$ to the quadratic polynomial equation with real coefficients $a \stackrel{o}{\varphi}+b \stackrel{o}{\varphi}+c=0$, the scators $\stackrel{o}{\varphi}_{\mathbb{S}^{1+2} \backslash \mathbb{S}^{1+1}}=f_{0} \pm f_{1} \check{\mathbf{e}}_{1} \pm f_{2} \check{\mathbf{e}}_{2}$ are also solutions to this equation.

Proof. The solution $\stackrel{o}{\varphi}_{\mathbb{S}^{1+2} \backslash \mathbb{S}^{1+1}}=f_{0}-f_{1} \check{\mathbf{e}}_{1}-f_{2} \check{\mathbf{e}}_{2}$ can be obtained following the usual complex proof of the conjugate polynomial equation. To prove that the sign of any one director can be changed, consider one such component. The $\check{\mathbf{e}}_{1}$ component of the squared scator can only cancel out with the $\check{\mathbf{e}}_{1}$ component of the linear scator term since the polynomial coefficients are real, thus

$$
a \cdot 2 f_{0} f_{1}\left(1-\frac{f_{1}^{2}}{f_{0}^{2}}\right) \check{\mathbf{e}}_{1}=b f_{1} \check{\mathbf{e}}_{1} \quad \Rightarrow \quad 2 f_{0}\left(1-\frac{f_{1}^{2}}{f_{0}^{2}}\right)=\frac{b}{a}
$$

If this result is satisfied for $f_{1}$ it also holds for $-f_{1}$. A similar argument is true for the $\check{\mathbf{e}}_{2}$ component. 


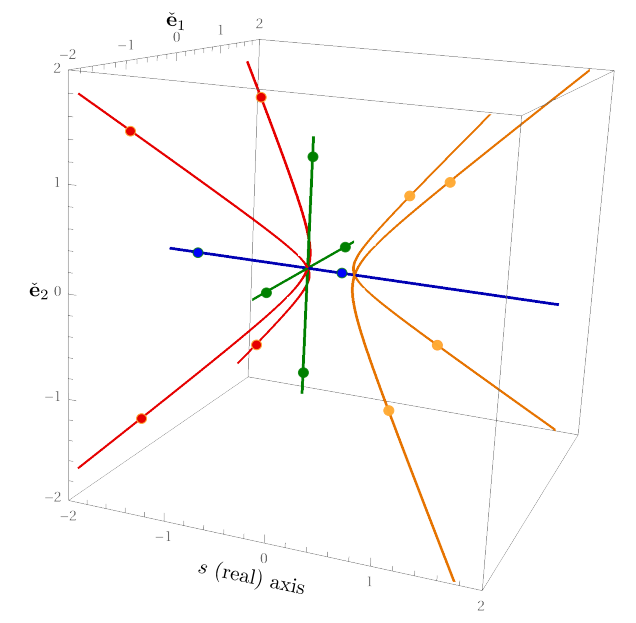

Fig. 3. Image of the polynomial roots for $a=b=1$ with $c$ as a parameter in the \pm 2 interval in each axis, $\stackrel{o}{f}(1,1, c)=\stackrel{o}{\varphi}^{2}+\stackrel{o}{\varphi}+c$. Real solutions in blue, complex like solutions in green and hypercomplex solutions for $4 a c>0$ in red and $4 a c<0$ in orange. Roots for $c=1$ (red and green dots) and $c=-1$ (blue and orange dots)

The hypercomplex roots clearly have the same magnitude (4), since $\|\stackrel{o}{\varphi}\|$ depends only on the square of the scator coefficients. The magnitude of the hypercomplex roots $(7 \mathrm{a})$, noticing that the squared ratio of director over scalar components is $\frac{f_{1}^{2}}{f_{0}^{2}}=\frac{4 a c-b^{2}}{4 a c+b^{2}}$, is given by

$$
\left\|\stackrel{o}{\varphi}_{\mathbb{S}^{1+2} \backslash \mathbb{S}^{1+1}}\right\|=2\left|\frac{c}{b}\right|
$$

The solutions as a function of $a$, depicted in figure 4, lie in an isometric surface since the above equation is independent of $a$. The elliptic scators isometric surface, named a cusphere, is illustrated in Figs. 5 and 6 in [9]. The teal curves in figure 4, are scator isometric ellipses lying on one of the two planes at $\pm 45^{\circ}$ with respect to the $\check{\mathbf{e}}_{1}, \check{\mathbf{e}}_{2}$ director axes. The projection of these ellipses in the $s, \check{\mathbf{e}}_{1}$ and $s$, $\check{\mathbf{e}}_{2}$ planes are actually circles with unit radius centered at -1 , as we shall now see.

Consider the change of variable $u=\frac{1}{4 a}$, the hypercomplex solutions (7a), can then be written as

$$
\stackrel{o}{\varphi}_{\mathbb{S}^{1+2} \backslash \mathbb{S}^{1+1}}=-\frac{c}{b}-b u \pm \sqrt{\frac{c^{2}}{b^{2}}-b^{2} u^{2}} \check{\mathbf{e}}_{1} \pm \sqrt{\frac{c^{2}}{b^{2}}-b^{2} u^{2}} \check{\mathbf{e}}_{2}
$$

Let $c$ and $b$ be constants. The above expression is then recognized as the parametric representation of the circle equation $x^{2}+y^{2}=\frac{c^{2}}{b^{2}}$, shifted by $\frac{c}{b}$ in the negative scalar axis, where $x=-b u$ and $y=\sqrt{\frac{c^{2}}{b^{2}}-b^{2} u^{2}}$ is the director coefficient in the $\check{\mathbf{e}}_{1}$ or the $\check{\mathbf{e}}_{2}$ axes. These circle projections in the $s, \check{\mathbf{e}}_{1}$ and $s, \check{\mathbf{e}}_{2}$ planes, exclude the points when the circles cross the scalar axis, i.e. $c^{2}=b^{4} u^{2}$. If $b$ and $u$ are considered constant, a parametric representation of the hyperbolic equation $x^{2}-y^{2}=b^{2} u^{2}$, is obtained, this time shifted by $-b u$ in the scalar axis, where $x=-\frac{c}{b}$ and $y$ is the director coefficient in either axis. These curves are plotted in red in figure 4 , the asymptotes lie at $\pm 45^{\circ}$ in the $s$, $\check{\mathbf{e}}_{1}$ or $s, \check{\mathbf{e}}_{2}$ planes. The vertices are located at 0 and $-2 b u=-\frac{b}{2 a}$, but these two points are not solutions to $\stackrel{o}{\varphi}_{\mathbb{S}^{1+2}} \backslash \mathbb{S}^{1+1}$, since $\left|\frac{c}{u}\right|=|4 a c|$ must be greater than $b^{2}$. If $c$ and $u$ are constant, the parametric equation (13) as a function of $b$, is no longer a conic curve.

The limit of the hypercomplex, real and complex like roots when $4 a c \rightarrow b^{2}$ is the same,

$$
\lim _{4 a c \rightarrow b^{2}}\left(\stackrel{o}{\varphi}_{\mathrm{sol}} \mathbb{S}^{1+2} \backslash \mathbb{S}^{1+1}\right)=\lim _{4 a c \rightarrow b^{2}}\left(\stackrel{o}{\varphi_{\mathrm{sol}} \mathbb{S}^{1+0}}\right)=\lim _{4 a c \rightarrow b^{2}} \stackrel{o}{\varphi}_{\mathrm{sol} \mathbb{S}_{1,2}^{1+1}}=-\frac{b}{2 a}
$$

Hypercomplex (red), real (blue) and complex like (green) curves approach $-\frac{b}{2 a}=-\frac{1}{2}$ in the Figure 3. 


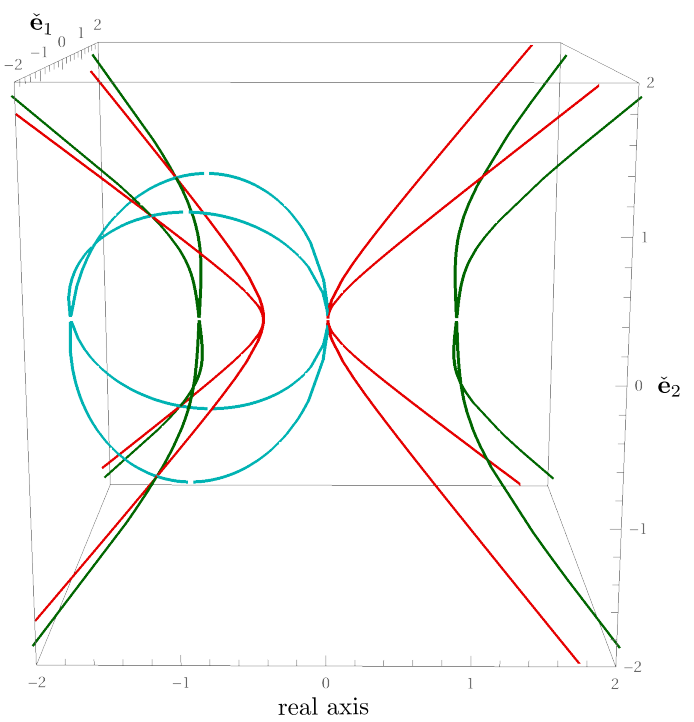

Fig. 4. Hypercomplex roots of the scator function, $\stackrel{o}{f}(a, b, c)=a \stackrel{o}{\varphi}^{2}+b \stackrel{o}{\varphi}+c$ for $|4 a c|>b^{2}$ in the \pm 2 interval in each direction. $a=b=1$ with $c$ as a parameter in red (red and orange in figure 3); $a=c=1$ with $b$ as a parameter in green; $b=c=1$ with $a$ as a parameter in teal. All curves lie in planes that are at $\pm 45^{\circ}$ with respect to the $\check{\mathbf{e}}_{1}, \check{\mathbf{e}}_{2}$ axes.

The limit of the hypercomplex and real roots when $4 a c \rightarrow-b^{2}$ are

$$
\lim _{4 a c \rightarrow-b^{2}}\left(\begin{array}{l}
o \\
\varphi_{\text {sol }}
\end{array} \mathbb{S}^{1+2} \backslash \mathbb{S}^{1+1}\right)=0 \quad \text { and } \quad \lim _{4 a c \rightarrow-b^{2}}\left(\stackrel{o}{\varphi_{\text {sol }} \mathbb{S}^{1+0}}\right)=-\frac{b}{2 a}(1 \pm \sqrt{2})
$$

However, it should be noted that the hypercomplex root function domain excludes $4 a c=-b^{2}$, so that 0 is not a root of the polynomial. In Figure 3, the hypercomplex curve (orange) does not intersect the real curve (blue) at zero. However, as $4 a c$ approaches $-b^{2}$ from the negative real axis, all three hypercomplex scator coefficients become infinitesimal. Nonetheless, from (12), the magnitude of the scator in this limit is $\lim _{4 a c \rightarrow-b^{2}}||{\stackrel{o}{\varphi^{1+2}}}_{\mathbb{S}^{1+1}} \|=2\left|\frac{c}{b}\right|=\left|\frac{b}{2 a}\right|$.

\subsection{Roots of Minus One}

The square roots of minus one have been studied in quaternions [10,11], complexified quaternions [12], split quaternions [13] and more generally in Clifford algebras [14]. In scator algebra, the hypercomplex roots tend to infinity as $b$ tends to zero, the green curves in Figure 4 are then asymptotic to $\pm 45^{\circ}$ lines. If $b^{2} \ll|4 a c|$, the binomial expansion of the roots to first non-vanishing order is

$$
\stackrel{o}{\varphi}_{\mathbb{S}^{1+2} \backslash \mathbb{S}^{1+1}} \approx-\frac{c}{b}(1+\delta) \pm \frac{c}{b}\left(1-\frac{1}{2} \delta^{2}\right) \check{\mathbf{e}}_{1} \pm \frac{c}{b}\left(1-\frac{1}{2} \delta^{2}\right) \check{\mathbf{e}}_{2}
$$

where $\delta=\frac{b^{2}}{4 a c}$. In the limit when $b \rightarrow 0$, each component diverges so that the solution diverges. The hypercomplex solutions in this limit become $\stackrel{o}{\varphi}_{\mathbb{S}^{1+2} \backslash \mathbb{S}^{1+1}}=-\frac{c}{b} \pm \frac{c}{b} \check{\mathbf{e}}_{1} \pm \frac{c}{b} \check{\mathbf{e}}_{2}$, but recall that scators whose three components have equal absolute value are square nilpotent [6, Lemma 1]. This result can also be readily seen from (11), for $f_{0}=f_{1}=f_{2}$. The quadratic polynomial leading term is thus zero $a \cdot o_{\mathbb{S}^{1+2} \backslash \mathbb{S}^{1+1}}^{2}=0$, and the polynomial equation becomes $b^{\circ} \stackrel{o}{\varphi}+c=0$. However, no scator in $\mathbb{S}^{1+2} \backslash \mathbb{S}^{1+1}$ satisfies this equation since $b, c$ are real. Therefore, there are no hypercomplex square roots in $\mathbb{S}^{1+2} \backslash \mathbb{S}^{1+1}$ of a real number, be it positive or negative. If $b=0$, then the only possible polynomial roots are

$$
\stackrel{o}{\varphi}_{\mathbb{S}_{1}^{1+1}}= \pm \sqrt{\frac{c}{a}} \check{\mathbf{e}}_{1}, \quad \stackrel{o}{\varphi}_{\mathbb{S}_{2}^{1+1}} \pm \sqrt{\frac{c}{a}} \check{\mathbf{e}}_{2}, \text { if } a c>0
$$

For $a=c$, the roots of -1 in $\mathbb{S}^{1+2}$ are thus $\pm \check{\mathbf{e}}_{1}$ and $\pm \check{\mathbf{e}}_{2}$. 


\section{Conclusions}

The quadratic polynomial $a x^{2}+b x+c=0, x=\stackrel{o}{\varphi} \in \mathbb{S}^{1+2}$ in the imaginary scator set with real coefficients $a, b, c$, has a finite number of roots. In sharp contrast, an infinite number of roots to the quadratic equation are encountered in quaternions and more generally in Clifford algebras. In the $\mathbb{S}^{1+2}$ scator set:

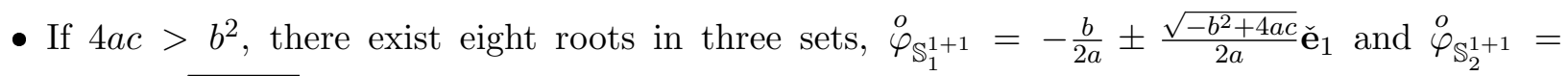
$-\frac{b}{2 a} \pm \frac{\sqrt{-b^{2}+4 a c}}{2 a} \check{\mathbf{e}}_{2}$ give two sets of two roots that are akin to the complex roots but the hyperimaginary units are now $\check{\mathbf{e}}_{1}$ and $\check{\mathbf{e}}_{2}$ instead of $i$. Four hypercomplex roots ${\stackrel{o}{\mathbb{S}^{1+2}} \backslash \mathbb{S}^{1+1}}=$ $-\frac{4 a c+b^{2}}{4 a b} \pm \sqrt{\frac{(4 a c)^{2}-\left(b^{2}\right)^{2}}{16 a^{2} b^{2}}} \check{\mathbf{e}}_{1} \pm \sqrt{\frac{(4 a c)^{2}-\left(b^{2}\right)^{2}}{16 a^{2} b^{2}}} \check{\mathbf{e}}_{2}$, that involve non zero components in both hypercomplex axes.

- If $|4 a c| \leq b^{2}$ there exist two real roots, $\stackrel{o}{\varphi}_{\mathbb{S}^{1+0}}=-\frac{b}{2 a} \pm \frac{\sqrt{b^{2}-4 a c}}{2 a}$ identical to the roots in the real set.

- If $4 a c<b^{2}$ and $|4 a c|>b^{2}$, there exist six roots in two sets, two real roots $\stackrel{o}{\varphi}_{\mathbb{S}^{1+0}}$ and four hypercomplex roots $\stackrel{o}{\varphi}_{\mathbb{S}^{1+2}} \backslash \mathbb{S}^{1+1}$.

Hypercomplex roots always come in sets of four in $\mathbb{S}^{1+2}$, in as much as complex roots come in sets of two in $\mathbb{C}$. Hypercomplex roots coexist with the real or complex like roots, in contrast with roots in the complex set, where the roots are either real xor complex. If $b$, the polynomial linear coefficient vanishes, the hypercomplex roots become square nilpotent so that the only roots of minus one are $\pm \check{\mathbf{e}}_{1}$ and $\pm \check{\mathbf{e}}_{2}$. Arbitrary integer powers of scators and nilpotent elements are discussed in [15]. The $\mathbb{S}^{1+2}$ scator roots can be visualized geometrically in a three dimensional space, where the scalar (real) axis and the two hypercomplex axes are drawn in orthogonal directions.

\section{Author Contributions}

The author read and approved the last version of the manuscript.

\section{Conflicts of Interest}

The author declares no conflict of interest.

\section{References}

[1] L. Huang, W. So, Quadratic Formulas for Quaternions, Applied Mathematics Letters 15(5) (2002) $533-540$.

[2] E. Macias-Virgos, M. Pereira-Saez, On the Quaternionic Quadratic Equation $x a x+b x+x c+d=0$, Advances in Applied Clifford Algebras 29(81) (2019) 1-13.

[3] M. Fernandez-Guasti, Associativity in Scator Algebra and the Quantum Wavefunction Collapse, Universal Journal of Mathematics and Applications 1(2) (2018) 80-88.

[4] M. Fernandez-Guasti, A non-distributive Extension of Complex Numbers to Higher Dimensions, Advances in Applied Clifford Algebras 25 (2015) 829-849.

[5] M. Fernandez-Guasti, Composition of Velocities in a Scator Deformed Lorentz Metric, European Physical Journal - Plus 135 (2020) 542.

[6] M. Fernandez-Guasti, Imaginary Scators Bound Set under the Iterated Quadratic Mapping In 1+2 Dimensional Parameter Space, International Journal of Bifurcation and Chaos 26(1) (2016) 1630002. 
[7] J. L. Cieslinski, D. Zhalukevich, Explicit Formulas for All Scator Holomorphic Functions in the (1+2)-Dimensional Case, Symmetry 12(9) (2020) 1-6.

[8] N. Bourbaki, Algebra I, Elements of Mathematics, Springer Verlag, 2007.

[9] M. Fernandez-Guasti, Differential Quotients in Elliptic Scator Algebra, Mathematical Methods in the Applied Sciences 41(12) (2018) 4827-4840.

[10] I. Niven, The Roots of a Quaternion, The American Mathematical Monthly 49(6) (1942) 386-388.

[11] L. Brand, The Roots of a Quaternion, The American Mathematical Monthly 49(8) (1942) 519520 .

[12] S. J. Sangwine, Biquaternion (Complexified Quaternion) roots of -1, Advances in Applied Clifford Algebras 16 (2006) 63-68.

[13] M. Ozdemir, The roots of a Split Quaternion, Applied Mathematics Letters 22(2) (2009) 258-263.

[14] E. Hitzer, R. Ablamowicz, Geometric Roots of -1 in Clifford Algebras $C_{p, q}$ with $p+q \leq 4$, Advances in Applied Clifford Algebras 21 (2010) 121-144.

[15] M. Fernandez-Guasti, Powers of Elliptic Scator Numbers, Preprints (2021) doi: 10.20944/preprints202108.0572.v1. 